\title{
TARDOC: remise de la version 1.2 au Conseil fédéral
}

\author{
Christian Oeschgera, Patrick Müller ${ }^{b}$ \\ a Expert, division Médecine et tarifs ambulatoires; ${ }^{b}$ chef de la division Médecine et tarifs ambulatoires FMH
}

En 2019, la FMH et curafutura ont soumis la version 1.0 du TARDOC au Conseil fédéral dans le but de remplacer le TARMED, devenu obsolète. Après examen, l'OFSP a estimé que le TARDOC pouvait être approuvé moyennant quelques adaptations. Ce qui a été fait dans la version 1.2, remise fin mars au Conseil fédéral. La FMH, curafutura et la CTM ont ainsi tout mis en œuvre pour que le TARDOC soit à présent rapidement approuvé et qu'il puisse entrer en vigueur le $1^{\text {er }}$ janvier 2022.

Le 20 novembre 2020, soit 500 jours après le dépôt de la demande d'approbation du TARDOC, l'Office fédéral de la santé publique (OFSP) a fait parvenir le rapport d'évaluation tant attendu aux partenaires tarifaires curafutura et FMH. Dans son rapport, l'OFSP conclut que le TARDOC peut être approuvé moyennant quelques adaptations. Le Conseil fédéral n'a en revanche pas encore pris de décision à ce sujet.

Pour la FMH, les points essentiels critiqués par l'OFSP sont les suivants:

- L'OFSP considère que le revenu de référence sur lequel repose la prestation médicale (PM) est trop élevé. Il critique en particulier le fait que le calcul se fonde non seulement sur les revenus des chefs de clinique, mais aussi sur ceux des médecins adjoints et des médecins-chefs.

- La durée quotidienne de travail prise en compte pour calculer la PM dans le modèle de coûts a aussi été critiquée par l'OFSP, qui propose de la fixer à 11,4 heures. La proposition des partenaires tarifaires est de 9,2 heures.

- Dans le modèle de coûts KOREG, c'est la représentativité des données provenant de l'étude permanente sur les coûts (RoKo) qui est remise en question, en plus d'autres points critiqués tels que l'architecture du modèle par exemple.

- Dans le modèle de coûts INFRA, ce sont notamment les coûts de construction, les coûts des appareils, les frais de personnel et leurs bases de données qui sont critiqués.

- Quant au concept de neutralité des coûts, l'OFSP souligne en particulier que la phase de monitorage est trop courte.

L'OFSP critique également le concept de valeur intrinsèque ainsi que d'autres points tels que la combinaison de prestations au temps et à l'acte, les limitations en partie moins strictes que celles fixées par le Conseil fédéral dans le TARMED, les valeurs intrinsèques élargies dans certains domaines ou les positions pour les consultations pressantes et d'urgence.

\section{Table ronde du conseiller fédéral A. Ber- set avec tous les partenaires tarifaires}

Après l'envoi du rapport d'évaluation du TARDOC en novembre à la FMH et à curafutura, le conseiller fédéral Alain Berset a convoqué le 3 décembre 2020 une table ronde à laquelle étaient invités tous les partenaires tarifaires (santésuisse, $\mathrm{H}+$, curafutura, FMH) ainsi que des représentants de la Conférence des directrices et directeurs cantonaux de la santé (CDS). A cette occasion, A. Berset a communiqué que deux projets avaient été soumis à l'approbation du Conseil fédéral. D'une part, la structure tarifaire TARDOC déposée par les partenaires tarifaires FMH, curafutura et SWICA et, d'autre part, un projet avec des forfaits facultatifs déposé par santésuisse et la FMCH.

Après de longues négociations, une déclaration d'intention relative à la collaboration et à l'approbation d'une organisation commune pour le tarif ambulatoire a été signée début mars par tous les partenaires tarifaires. Le délai prévu - «aussi rapidement que possible mais fin 2021 au plus tard» - pour la remise du TARDOC remanié selon les recommandations de l'OFSP est cependant trop tardif pour curafutura et la FMH. La FMH, curafutura et la Commission des tarifs médicaux LAA (CTM) souhaitent toujours que le TARDOC soit approuvé rapidement après le dépôt des modifications et qu'il entre en vigueur le $1^{\text {er }}$ janvier 2022. 


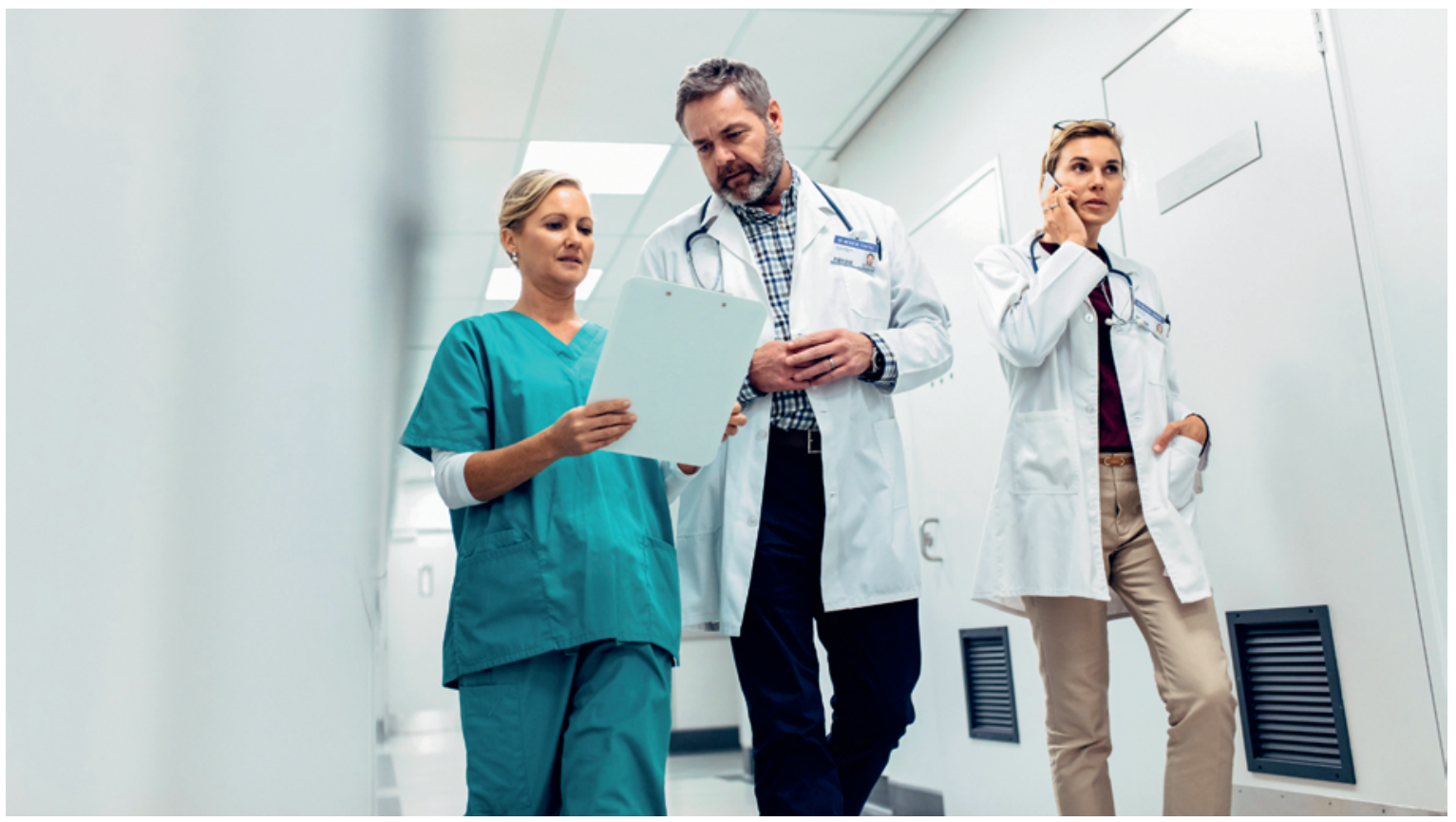

L'objectif de la FMH et de curafutura, mais aussi de la CTM, est que le TARDOC entre en vigueur en 2022 et remplace le TARMED qui ne peut plus être révisé.

\section{Remise du TARDOC 1.2 fin mars 2021}

Les points critiqués par l'OFSP ont été systématiquement pris en compte et traités entre décembre et début mars par le siège d'ats-tms SA et les partenaires tarifaires (FMH, curafutura et CTM). Lorsque nécessaire, la FMH a pris contact avec les sociétés de discipline concernées. L'objectif était de déposer la version 1.2, avec une prise de position complète en guise de réponse au rapport d'évaluation de l'OFSP, auprès de ce dernier. Le dépôt, fin mars 2021, des deux concepts révisés (neutralité des coûts et valeur intrinsèque) et de la prise de position des partenaires tarifaires d'ats-tms SA impliqués doit à présent permettre d'obtenir rapidement l'approbation du TARDOC par le Conseil fédéral. Parmi les thèmes importants pour toutes les sociétés de médecine affiliées à la FMH figurent le revenu de référence, le temps de travail annuel des médecins, le concept de neutralité des coûts et le concept de valeur intrinsèque (droits acquis). Ces points ont fait l'objet de discussions entre la FMH, curafutura et la CTM au cours des dernières semaines. Ci-après sont résumées les principales modifications.

\section{Prestation médicale (revenu de référence et temps de travail annuel)}

L'OFSP a critiqué d'une part la composition du revenu de référence. En effet, il estime que les salaires des chefs de clinique, et non pas une moyenne pondérée des salaires des médecins-chefs, médecins adjoints et chefs de clinique (comme c'est le cas dans l'étude sur le revenu de référence), doivent être utilisés à titre de comparaison afin de respecter le principe d'opportunité. D’autre part, il a critiqué la durée quotidienne de travail de 9,2 heures et exigé que celle-ci soit relevée à 11,4 heures.

Pour la FMH, il est prioritaire de défendre le taux de coûts de la PM. C'est pourquoi elle a mandaté deux expertises, en concertation avec les partenaires tarifaires. Le Prof. Thomas Geiser, spécialiste du droit du travail, a été mandaté pour examiner l'exigence d'augmenter la durée quotidienne de travail à 11,4 heures. Dans son avis de droit, le Prof. Geiser constate que l'exigence de l'OFSP est contraire à la loi et qu'elle devrait se fonder sur un maximum de 50 heures hebdomadaires (conformément à la loi sur le travail). La FMH et curafutura maintiennent donc la durée de travail à 9,2 heures par jour.

L'Institut d'économie de la santé de Winterthour (WIG) de la Haute école des sciences appliquées de Zurich (ZHAW) a également pris position sur l'exigence de l'OFSP d'adapter le revenu de référence. Le WIG conteste l'exigence de l'OFSP et confirme les conclusions et paramètres qu'il avait établis dans l'étude sur le revenu de référence. Les partenaires tarifaires maintiennent donc aussi leur position concernant l'étude et le niveau du revenu de référence. 


\section{Concept de neutralité des coûts}

Dans son rapport d'évaluation, l'OFSP a demandé qu'une neutralité dynamique des coûts soit mise en œuvre pour une durée d'au moins trois ans. Les partenaires tarifaires ont donc révisé certaines parties du concept de neutralité des coûts sur la base du rapport d'évaluation. Ils ont convenu que la phase de mesure et de pilotage soit prolongée de 18 à 30 mois. On pourra ainsi mesurer et piloter les effets du changement de modèle tarifaire sur une période plus longue (2,5 ans). Les partenaires tarifaires estiment satisfaire aux conditions visées à l'art. 59c, al. 1, let. c, OAMal.

\section{Concept de valeur intrinsèque}

L'OFSP a totalement remis en question la solution prévue pour le maintien des droits acquis et vivement critiqué la durée prévue de 10 ans. Après négociation, la FMH et curafutura ont réduit la durée du maintien des droits acquis de 10 à 6 ans. En contrepartie, la condition pour les faire valoir sur les prestations facturées est adaptée à trois ans avant l'introduction du TARDOC.

\section{Prochaines étapes}

L'objectif de la FMH et de curafutura, mais aussi de la CTM, est que le TARDOC entre en vigueur en 2022 et remplace le TARMED qui ne peut plus être révisé.

Dès sa mise en application, le TARDOC sera révisé chaque année, contrairement au tarif actuellement en vigueur. Pour cela, la FMH, curafutura et la CTM ont fondé l'organisation tarifaire commune ats-tms SA, qui a pour tâche de poursuivre le développement du TARDOC sur mandat des sociétaires. Les conditions nécessaires ont déjà été établies au sein des organes ad hoc: le principe de l'unanimité, notamment, ne s'appliquera plus pour les décisions prises par le conseil d'administration d'ats-tms SA. De cette façon, le développement de la structure tarifaire pourra être poursuivi en continu en faveur de toutes les parties.

Divers projets ont ainsi déjà été entamés au sein d'atstms SA, par exemple le projet "Granularité du TARDOC» qui est en cours. Des interviews ont été menées pour cela avec différentes sociétés de discipline. Il s'agit d'examiner dans quelle mesure le TARDOC pourrait être encore simplifié en combinant le temps nécessaire à la préparation et à la finition, le temps de transition et d'examen, ainsi que la documentation et les rapports. L'objectif est de simplifier la structure tarifaire sans violer les principes d'adéquation et de transparence. D'autres travaux se poursuivent en parallèle dans les domaines du contrôle de la plausibilité des minutages, de la vérification des modèles de coûts et de la prise en charge et surveillance paramédicales. Ces derniers jours, la nouvelle commission d'interprétation et les processus relatifs à la future procédure de demande pour le développement du tarif ont été mis sur les rails.

\section{Crédits photo}

Ammentorp | Dreamstime.com (photo prétexte)

\section{FMH}

Division Médecine et tarifs ambulatoires

Baslerstrasse 47 CH-4600 Olten

Tél. 0313591230

Fax 0313591238

tarife.ambulant[at]fmh.ch

\section{Rappel des faits}

La FMH et curafutura ont soumis la version 1.0 du TARDOC au Conseil fédéral à l'été 2019. La Commission des tarifs médicaux LAA (CTM) n'a pas participé à la remise du tarif, mais fait figure de partenaire du TARDOC pour les domaines de I'assuranceaccidents, de l'assurance-invalidité et de I'assurance militaire. L'Office fédéral de la santé publique (OFSP) a ensuite procédé à l'examen de fond du tarif.

Dans le même temps, le conseiller fédéral Alain Berset signalait à la FMH et curafutura qu'il considérait que leTARDOC ne pouvait pas être approuvé dans sa version 1.0. II a souligné que les assureurs de curafutura, à savoir CSS, Helsana, Sanitas et KPT, ne représentaient pas une majorité parmi les assureurs et qu'un grand nombre d'assurés n'étaient pas représentés en raison de la non-participation des assureurs de santésuisse. Alain Berset a aussi regretté que la FMH et curafutura aient déposé deux concepts différents pour la transition neutre en termes de coûts deTARMED àTARDOC.

Suite à cela, la FMH et curafutura ont déposé à l'été 2020 une nouvelle version (1.1) duTARDOC. Pour la première fois, les deux partenaires ont aussi remis un concept de neutralité des coûts négocié ensemble. Le nouveau concept prévoit un «facteur externe" qui fait partie intégrante de la structure tarifaire, mais $\mathrm{n}^{\prime}$ est pas facturé via les points tarifaires. A ce sujet, nous vous recommandons de lire l'article paru dans le Bulletin des médecins suisses du 15 juillet 2020: "L'introduction duTARDOC ne doit entraîner aucun coût supplémentaire» [1].

Grâce au concept commun de neutralité des coûts et à la structure tarifaire uniforme qui en résulte, la FMH et curafutura ont répondu à deux exigences du Conseil fédéral. L'introduction du TARDOC ne doit entraîner aucun coût supplémentaire. Le $1^{\text {er }}$ mai 2020, SWICA (la cinquième assurance-maladie de Suisse) a décidé de soutenir également le TARDOC et de faire partie de la solution. Avec le soutien de SWICA au TARDOC, ce sont donc $53 \%$ des assurés qui sont derrière le tarif, ainsi désormais soutenu par une majorité, tant du côté des fournisseurs de prestations que des assureurs.

1 Oeschger C. L'introduction duTARDOC ne doit entraîner aucun coût supplémentaire. Bull Med Suisses. 2020;101(29-30):878-9. 